\title{
Iron Fortification in Parboiled Rice-A Rapid and Effective Tool for Delivering Iron Nutrition to Rice Consumers
}

\author{
Chanakan Prom-u-thai ${ }^{{ }^{*}}$, Longbin Huang ${ }^{2}$, Shu Fukai ${ }^{3}$, Benjavan Rerkasem ${ }^{1}$ \\ ${ }^{1}$ Agronomy Division, Department of Plant Science and Natural Resources, Faculty of Agriculture, Chiang Mai University, Chiang Mai, \\ Thailand; ${ }^{2}$ Center for Mined Land Rehabilitation, The University of Queensland, St Lucia, Australia; ${ }^{3}$ School of Land, Crop and Food Sci- \\ ence, The University of Queensland, St Lucia, Australia. \\ Email: chanakan15@hotmail.com
}

Received June $15^{\text {th }}, 2010$; revised August $13^{\text {th }}, 2010$; accepted January $17^{\text {th }}, 2011$.

\begin{abstract}
Parboiled rice production accounts for nearly half of the world's rice production. Its markets and consumer base are firmly established in South Asia and Africa where Fe-deficient populations are mostly concentrated. Our research group has pioneered the technology of Fe-fortification in parboiled rice and demonstrated its feasibility in significantly increasing Fe concentration in the endosperm (white rice) and its bioavailability in rice based diet. Fortification with Fe-EDTA during parboiling resulted in 10 to 50 folds increase in grain Fe concentration, depending on the grain properties among different rice varieties. However, the broken rice of Fe-fortified parboiled rice contained 5 times the Fe concentration of the full grain, which is often bought and consumed by people in low income category. The bioavailability of the fortified Fe is closely correlated with increasing Fe concentration in white rice $(r=0.90, p<0.01)$. The retention rates of the fortified $\mathrm{Fe}$ in the white rice range from $>50 \%$ to almost $100 \%$, despite repeated rinsing before cooking depending on rice varieties. Perls' Prussian blue staining and prolonged polishing showed that the in vitro Fe penetrated into the interior of the endosperm. Fortification at the rate up to $250 \mathrm{mg} \mathrm{Fe} \mathrm{kg}^{-1}$ paddy rice has no deleterious effects on appearance, color and sensory quality and overall acceptance by parboiled rice consumers. It increased

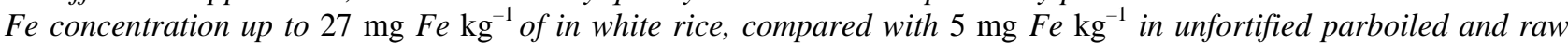
white rice. As a result, we can conclude that parboiled rice is a ready and effective tool for improving Fe nutrition of rice consumers in these regions.
\end{abstract}

Keywords: Fe Deficiency Anemia, Fe Fortified, Parboiled Rice, Fe Bioavailability

\section{Introduction}

Fortifying Fe in food has been suggested as a means to improve Fe level in food products and human diets [1,2]. This is especially among the population who consumes rice as the staple food and has limited access to Fe rich food such as animal products (meat, egg and milk) as in Asia and Africa where Fe deficiency anemia is a major wide spread problem $[3,4]$. Because of poor access to Fe-rich food ingredients, rice remains as a major source of dietary intake of mineral nutrients and vitamins among these countries which only supplying inadequate $\mathrm{Fe}$ in their daily meal [3]. However, Fe fortifying in raw rice grain has not been successful due to consumers' poor acceptance of the different appearance of the fortified grain mixed with normal grain and removal of the $\mathrm{Fe}$ fortified grains before cooking [5]. Surface coating of Fe by flour gel and/or cellulose polymer also tends to be washed away during rinsing (70\% - 100\%), a common practice in rice cooking [6,7]. In addition, fortification by surface coating also requires not only re-educated consumers, but new and special industrial infrastructure on a large scale, which are often economically unfeasible in developing countries.

The success and cost-effectiveness of in vitro Fe-fortification will depend on several key aspects 1) industrial feasibility and associated investment costs; 2) biological effectiveness of Fe fortified; and 3) consumers' acceptance. Parboiled rice production overcomes these constraints and offers an ideal vehicle for Fe fortification. Thus, it can be a promising strategy to improve both $\mathrm{Fe}$ density in parboiled rice grain and human nutrition among parboiled rice consumers. 


\section{Parboiling, a Ready Made Industrial Process for Iron Fortification}

Parboiled rice has been established for more than 20 centuries and it is produced in many part of the world such as India, Bangladesh, Pakistan, Myanmar, Malaysia, Sri Lanka, Guinea, South Africa, Italy, Spain, Thailand, Switzerland, USA and France [8]. The production of parboiled rice is currently about $>100$ million tons annually which is accounts for about half of the world rice crop, especially in Asia and Africa [9]. Many countries such as India (60\%) and Bangladesh (90\%) consume parboiled rice as the staple food [10]. Moreover, the infrastructures for production and market distribution have been well established in each location without major additional investment. Thailand, where practically no parboiled rice is consumed, exports 2 - 3 million tons of milled parboiled rice each year through the already existed market network to the countries in Middle East and Africa [11]. Processing facilities are also common in countries where parboiled rice is consumed such as India and Bangladesh. Our survey in Thailand found mills of varying degrees of sophistication, of which many are modern mills fitted with electronic eyes that screen out blacken or discolored grains. Briefly, the process of rice parboiling involves soaking the unhusked paddy rice, steaming and drying before milling to produce white parboiled rice, a preferred form among parboiled rice consumers [9]. The soaking time varies from 6 to 24 hours with varying water temperature depending on the technique in each mill, leaving the time channel for Fe fortification can be implemented by spiking suitable form and concentration of $\mathrm{Fe}$ in the soaking water under the optimal soaking conditions. As a result, this does not require major changes to the existing parboiling rice production process and infrastructure.

\section{Effectiveness of Iron Fortification by Parboiling}

Adding Fe to the paddy rice in the parboiling process significantly increases both total Fe concentration in the white rice grain and the bioavailable Fe fraction [12,13]. Under laboratory simulated parboiling conditions, Fe fortification increased $\mathrm{Fe}$ concentrations in milled rice for up to 50 folds, compared to the background level of 4

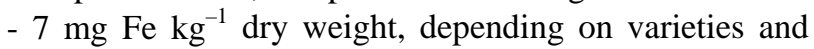
milling time (Figure 1). For example, after 60 seconds milling, the cultivars YRF 2 and Opus had the largest increment, with Fe concentration 20 times of those in unfortified raw rice. At $120 \mathrm{~s}$ milling time, the cultivar Opus, had as much as 50 times of the Fe concentration in the unfortified. Current findings indicate that the optimum condition for Fe fortification in parboiling process is soaking unhusked paddy rice at $60^{\circ} \mathrm{C}$ for $6 \mathrm{~h}$ in acidic pH of FeEDTA solution containing $250 \mathrm{mg} \mathrm{Fe} \mathrm{kg}^{-1}$ paddy rice. It is an effective technique in increasing Fe concentration in parboiled rice, which can increase Fe con-

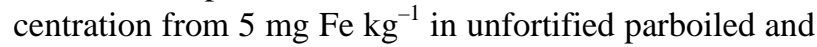
raw white rice, to the most desirable Fe concentration in white parboiled rice which is about $27 \mathrm{mg} \mathrm{Fe} \mathrm{kg}^{-1}$ (Ross Welch, personal communication), without adverse impact on rice cooking qualities such as color, flavor and textures [14]. However, the current finding observes that the concentration of $\mathrm{Fe}$ in white rice exponentially increased with increasing rate of Fe fortification with variation among rice varieties, indicating the different potential of Fe penetration into the inner layer of rice grain among rice varieties (Figure 2). For example, SPR 1 trends to have more potential in increasing of Fe concentration compared with CNT 1 when fortify at the same rate of $\mathrm{Fe}$.

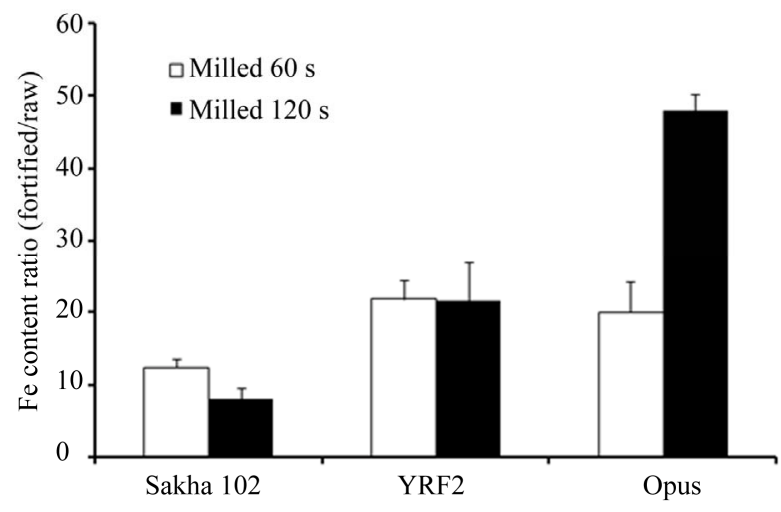

Figure 1. The ratio of Fe content in fortified rice grain to that of unfortified rice grain. The parboiled and raw grains were milled for 60 and 120 s, respectively. The bars re- present standard errors of corresponding means from 3 replicates (Prom-u-thai et al. 2008).

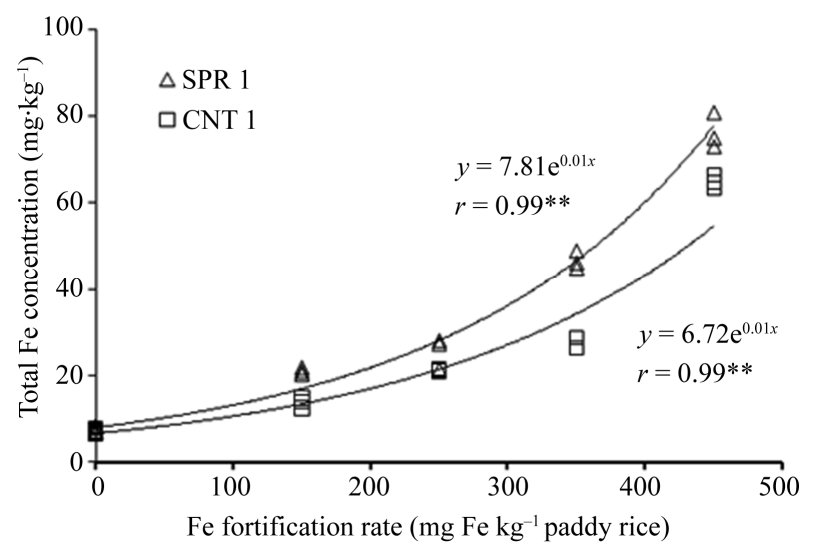

Figure 2. Relationship between Fe fortification rates and total Fe concentrations in white rice of Fe-fortified parboiled rice and unfortified and raw/parboiled rice in two rice varieties pooled $(n=18)$ (Prom-u-thai et al., inpress). 
However, the most significant finding is that the fortified Fe remained highly bioavailable and its bioavailability exhibited a close correlation within the total Fe concentration $(r=0.90, p<0.01)$ in the parboiled grains, indicating highly bioavailability of fortified $\mathrm{Fe}$ in parboiled rice [13]. Parboiled rice can be fortified to contain more bioavailable Fe than white bean, the standard for high Fe legume grain (Figure 3) [13]. The bioavailability of $\mathrm{Fe}$ in Fe fortified parboiled rice is correlate with total Fe concentration in all tested samples, establishing the bioavailable form of fortified Fe in parboiled white rice in human nutrition even after fortification through parboiling process [13]. Moreover, the study also established an easy and rapid method to evaluate the bioavailability of $\mathrm{Fe}$ in Fe-fortified parboiled rice by using dilute acid extraction as it was observed that it was closely correlates with the bioavailability of Fe established by an in vitro Caco-2 cell culture model [13].

\section{Fe Penetration and Retention in the Endosperm}

The fortified Fe effectively penetrated into the interior of the endosperm, which was clearly demonstrated by $\mathrm{Fe}$ localization staining with Perls’ Prussian blue (Figure 4). In the grains milled for $60 \mathrm{~s}$, unfortified raw rice grains only had a very low intensity of staining in the surface layer of the endosperm (Figure 4(a1) and (a2)), while in Fe-fortified and parboiled grain, a high intensity of staining was found in the outer layers (20\% - 30\% of the cross-section distance) of the endosperm of the fortified grains (Figures 4(b1) and (b2)) [12]. The results indicated that the distribution of the staining tended to diffuse through the dorsal region of the grain and gradually towards the opposite pole of the grain. From visual observation, the parboiling process achieved a significant penetration through the inner layers of the endosperm of the parboiled rice grains. The advantage of Fe penetration into the inner layer of the endosperm after fortification process ensure the retention of adequate Fe after polishing for optimum cooking qualities of rice grain, in contrast to the problem of significant Fe loss from milling of raw rice grains. This advantage also helps high Fe retention after rinsing prior to cooking, a common practice of rice consumers. The degree of Fe loss from rinsing in the Fe-fortified parboiled rice grains varied among cultivars and milling time (Figure 5). For example, the milled Fe-fortified rice retained $45 \%$ - 96\% Fe in the grains milled for $60 \mathrm{~s}$ and $20 \%$ - $98 \%$ in the grain milled for $120 \mathrm{~s}$ after rinsing.

\section{Fe Richness in Broken Parboiled Rice Fortified with Fe}

Broken rice is an undesirable in rice milling which as it lower the quality and price. The percentage of broken grain ( $<3 / 4$ to grain length) has negative impact on the price. For example, during the first half of March 2009 the price per ton of $100 \%$ parboiled rice (no broken) was quoted at US\$ 664, dropping to US\$ 479 with just 5\% broken grain and US\$ 336 per ton for rice sold as broken (Thai Rice Exporters Association 2009). Broken rice costs

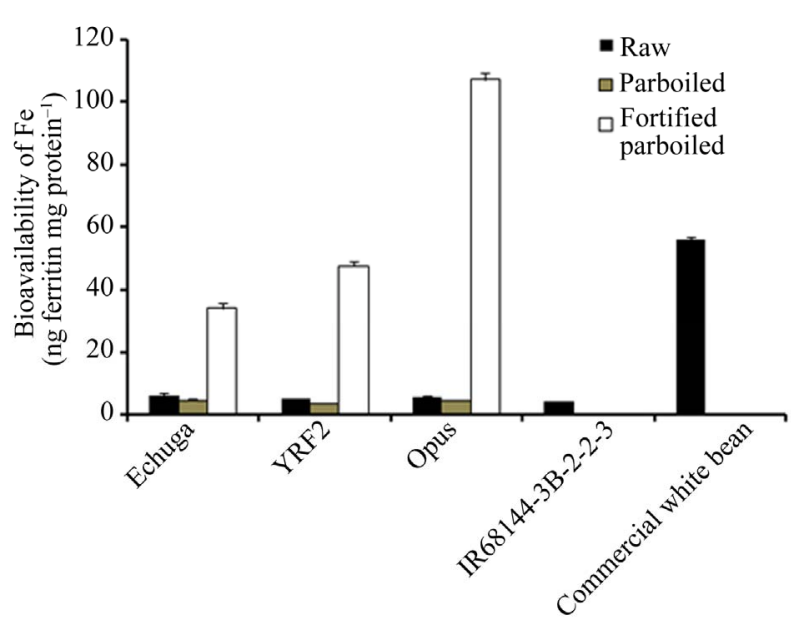

Figure 3. Bioavailability of Fe from digests of rice samples in 3 cultivars after milling for $120 \mathrm{~s}$, IR68144-2B-3-2-2, and a commercial US white bean were included. Values are mean \pm SEM $(n=3)$ (Prom-u-thai et al. 2009).
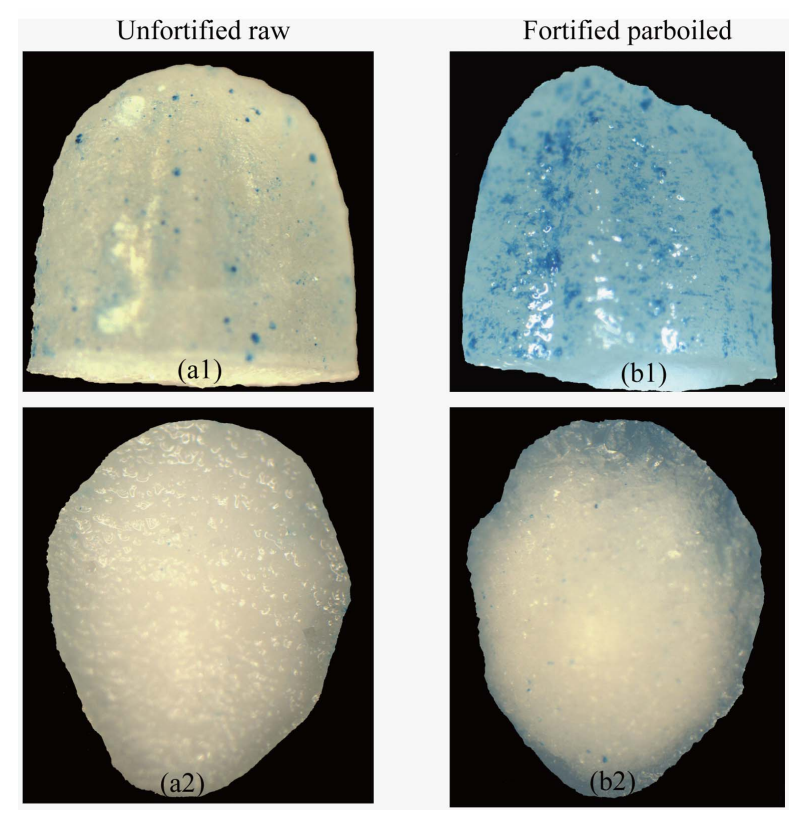

Figure 4. Stereo-micrographs of unfortified (a1, a2) and fortified (b1, b2) rice grains (cv. Opus), which were milled rice for $60 \mathrm{~s}$. The grains were cut transversely across the middle plane of the grain and were stained with Perls Prussian blue (side views - a1, b1; and top view - a2, b2). All scale bar $=1 \mathrm{~mm}$. The intensity of staining represented the relative density of Fe in the grains (Prom-u-thai et al. 2008). 


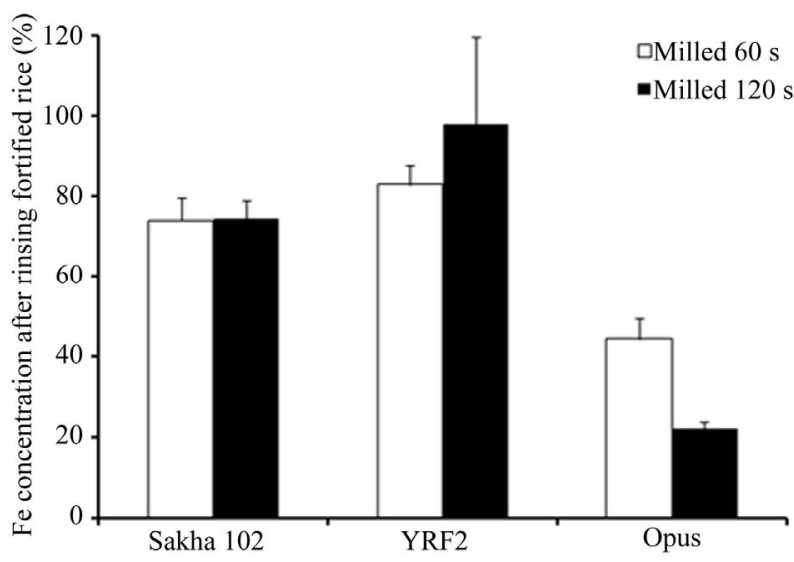

Figure 5. Iron retention rate (as \% of the un-rinsed) after rinsing (simulating rice washing) in the Fe-fortified parboiled rice grains milled for 60 and $120 \mathrm{~s}$, respectively, in the 3 rice cultivars tested (Prom-u-thai et al. 2008).

only $25 \%-50 \%$ of the $100 \%$ full grain rice and is preferred by lower income consumers for cost-saving. Because of its very low price compared with rice that is mostly or all whole grain, broken rice usually ends up as raw material in processing industry from rice flour to noodle and various snacks [15]. Broken rice is also the main staple for consumers with low income in many developing countries. Importers in Africa commonly buy broken parboiled rice from Thailand for the low end market. So we examined the Fe fortification effectiveness and density in broken rice for this neediest sector of the population who happens to have the highest risk of Fe-deficiency anemia. The Fe content of broken rice in unfortified and Fe fortified parboiled rice (with 250 and

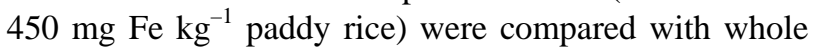
grain. The broken rice of unfortified and Fe-fortified parboiled rice at 250 and $450 \mathrm{mg} \mathrm{Fe} \mathrm{kg}^{-1}$ paddy rice, contained Fe concentrations ranging from $16-96 \mathrm{mg} \cdot \mathrm{kg}^{-1}$ white rice, while it was $7-18 \mathrm{mg} \cdot \mathrm{kg}^{-1}$ in the full grain (Figure 6). Unfortified, the Fe concentration was $7 \mathrm{mg} \cdot \mathrm{kg}^{-1}$ in whole grain compared with $16 \mathrm{mg} \cdot \mathrm{kg}^{-1}$ in the broken. For Fe fortified parboiled rice, Fe concentration in broken rice was 4 - 5 times of those in whole grain. This difference in Fe concentration between whole and broken grain of parboiled rice is likely to be composed of grain sections containing higher Fe. The fortified Fe tends to distributed in the tips of the grain that tend to break off during milling (Figure 7). In parboiled rice fortified with Fe, differential rates of Fe diffusion may occur in different sections of the rice grain during fortification. Therefore, this suggests that fortification of Fe during parboiling is a very promising and equitable approach for overcoming Fe-deficiency risks in economically advantaged and disadvantaged populations in the remote areas where broken rice is commonly consumes due to their low pur-

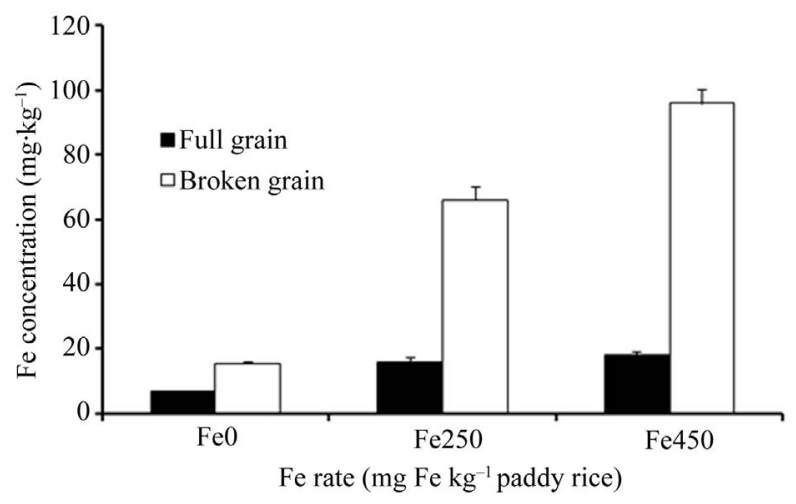

Figure 6. Fe concentrations in full and broken grains in cultivar CNT 1 of unfortified (Fe0) and fortified parboiled rice at 250 (Fe250) and 450 (Fe450) $\mathrm{mg} \mathrm{Fe} \mathrm{kg}^{-1}$ paddy rice.

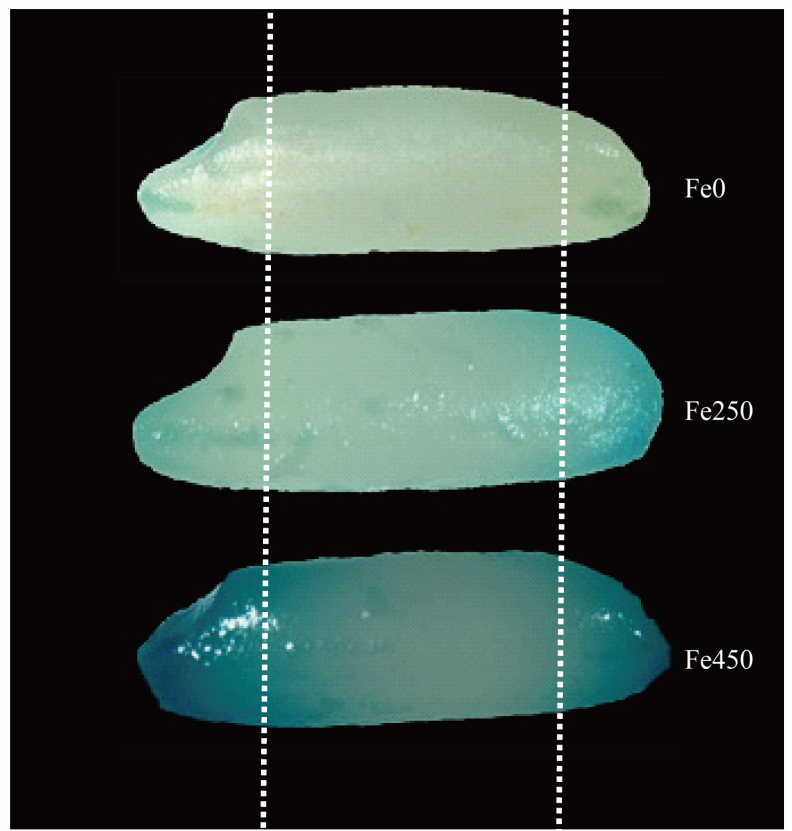

Figure 7. Stereo-micrographs showing position of rice grain that are likely to break off during milling process at both ends of rice grain (dash lines) in unfortified parboiled (above) and parboiled rice fortified with $\mathrm{Fe}$ at 250 (middle) and 450 (below) mg Fe $\mathrm{kg}^{-1}$ paddy rice (cv CNT 1), which were milled for $30 \mathrm{~s}$. The grains were stained with Perls Prussian blue. The intensity of staining represented the relative density of $\mathrm{Fe}$ in the grains.

chasing power.

\section{Consumer Acceptance and Remaining Issues}

The changes of pre-cooking quality, cooking quality and consumers' poor acceptance are critical reasons that have contributed to the lack of success of Fe fortification of raw rice $[5,16]$. These fundamental problems have not been found with $\mathrm{Fe}$ fortified parboiled rice at optimal $\mathrm{Fe}$ 
density. Two sensory panels, one in parboiled rice eating Bangladesh and one in Thailand where parboiled rice is hardly ever consumed, found the cooked parboiled rice fortified with appropriate rate of Fe to be indistinguishable from commercially available and local production parboiled rice. The sensory test among 19 panelists in Bangladesh gave an overall acceptability of $100 \%$ to the

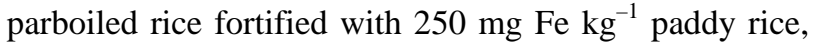
while $95 \%$ was given in unfortified parboiled rice. On the other hand, fortification with $450 \mathrm{mg} \mathrm{Fe} \mathrm{kg}{ }^{-1}$ paddy rice was given in lower acceptability (63\%), probable due to the changing of cooking quality such as color and/or flavor when fortified with high rate of Fe [14]. These initial results provide a great confidence of marketability and consumer acceptance of the Fe-fortified parboiled rice. However, the optimal rate of Fe fortification should be care as it is impact in consumer acceptability.

Further research needs to focus on appropriate cultivar-specific or grain-property-specific protocols of Fefortification during parboiling, such as soaking time, temperature, steaming and drying conditions. Physical and chemical properties of rice grain before fortification process may affect Fe entry and retention rate in the endosperm. A social survey on consumer's preference and opinion is also a significant topic to be investigated, particularly among the countries with high parboiled rice consumption.

\section{Conclusions}

With an established industry infrastructure and half of the world's rice production already parboiled, Fe-fortified parboiled rice offers a ready tool for significantly improving Fe nutrition in economically disadvantaged populations in south Asia and Africa, where Fe-deficiency anemia poses a great threat to human health and productivity. Fe-fortified parboiled rice can easily and rapidly reach rice consumers in these countries without the need to alter consumption habits of local populations and establishing new market network and access. The cheaper broken rice contains much more $\mathrm{Fe}$, which are mostly consumed by low income consumers. In summary, Fe-fortified parboiled rice offers highly cost-effective tool to reduce the incidences of Fe deficiency in developing countries within an immediate future if it is adopted by the current parboiled rice industry.

\section{Acknowledgements}

We acknowledge University of Queensland for postdoctoral research fund, McKnight Foundation, Thailand Research Fund, International Foundation for Science and Office of the Higher Education Commission, Thailand under the National Research University Project for fi- nancial support. We also thank Mr Tawatchai from Kasetsomboontanyakit Co., Ltd. and Mr Suradet Palawisut from Phitsanuloke Rice Research Center for providing seeds, Dr Sittichai Lordkaew from Multiple Cropping Center, Faculty of Agriculture, Chiang Mai University for Fe analysis assistant, Geoff Smith from Akzo Nobel Co. Ltd., Singapore for providing Ferrazone ${ }^{\circledR}$.

\section{REFERENCES}

[1] D. Moretti, T. C. Lee, M. B. Zimmermann, J. Nuessli and R. F. Hurrell, "Development and Evaluation of Iron-Fortified Extruded Rice Grains,” Journal of Food Science, Vol. 70, No. 5, 2005, pp. S330-S336. doi:10.1111/j.1365-2621.2005.tb09987.x

[2] D. Moretti, M. B. Zimmermann, S. Muthayya, P. Thankachan, T. C. Lee, A. V. Kurpad and R. F. Hurrell, "Extruded Rice Fortified with Micronized Ground Ferric Pyrophosphate Reduces Iron Deficiency in Indian Schoolchildren: A Double-Blind Randomized Controlled Trial," American Journal of Clinical Nutrition, Vol. 84, No. 4, 2006, pp. 822-829.

[3] B. O. Juliano, "Rice in Human Nutrition,” Food and Agriculture Organization of the United Nations, Rome, 1993.

[4] WHO, "The World Health Report 2002, Reducing Risks, Promoting Healthy Life,” World Health Organization, Geneva, 2002, pp. 1-168.

[5] J. D. Cook, M. B. Reddy, J. Burri, M. A. Juillerat and R. F. Hurrell, "The Influence of Different Cereal Grains on Iron Absorption from Infant Cereal Foods,” American Journal of Clinical Nutrition, Vol. 65, No. 4, 1997, pp. 964-969.

[6] A. Peil, F. Barrett, C. Rha and R. Langer, "Retention of Micronutrients by Polymer Coatings Used to Fortify Rice,” Journal of Food Science, Vol. 47, 1981, pp. 260-262. doi:10.1111/j.1365-2621.1982.tb11073.x

[7] V. Tulyathan, T. Mekjarutkul and S. Jongkaewwattana, "Iron Retention on Flour Gel-Coated Rice Grains and Its Storage Stability," Foodservice Research International, Vol. 15, 2005, pp. 147-156. doi:10.1111/j.1745-4506.2005.00005.x

[8] P. Pillaiyar, "Household Parboiling of Parboiled Rice," Kishan World, Vol. 8, 1981, pp. 20-21.

[9] K. R. Bhattacharya, "Parboiling of Rice," American Association of Cereal Chemists, Inc., St. Paul, 2004.

[10] N. H. Choudhury, "Parboiling and Consumer Demand of Parboiled Rice in South Asia," International Rice Research Institute, Manila, 1991.

[11] B. Rerkasem, "Having Your Rice and Eating It Too: A View of Thailand's Green Revolution,” Science Asia, Vol. 33, Supplement 1, 2007, pp. 75-80. doi:10.2306/scienceasia1513-1874.2007.33(s1).075

[12] C. Prom-u-thai, S. Fukai, D. I. Godwin, B. Rerkasem and L. Huang, "Iron-Fortified Parboiled Rice-A Novel Solution to High Iron Density in Rice-Based Diets,” Food 
Chemistry, Vol. 110, 2008, pp. 390-398. doi:10.1016/j.foodchem.2008.02.043

[13] C. Prom-u-thai, R. P. Glahn, Z. Cheng, S. Fukai, B. Rerkasem and L. Huang, "The Bioavailability of Iron Fortified in Whole Grain Parboiled Rice,” Food Chemistry, Vol. 112, No. 4, 2009, pp. 982-986. doi:10.1016/j.foodchem.2008.07.020

[14] C. Prom-u-thai, B. Rerkasem, S. Fukai and L. Huang, "Iron-Fortification and Parboiled Rice Quality: Appearance, Cooking Quality and Sensory Attributes,” Journal of the Science of Food and Agriculture, Vol. 89, No. 15,
2009, pp. 2565-2571. doi:10.1002/jsfa.3753

[15] M. Chitpan, V. Chavasit and R. Kongkachuichai, "Development of Fortified Dried Broken Rice as a Complementary Food,” Food and Nutrition Bulletin, Vol. 26, No. 4, 2005, pp. 376-384.

[16] Y. Li, L. L. Diosady and S. Jankowski, "Stability of Vitamin B1 in Ultra Rice in the Presence of Encapsulated Ferrous Fumarate,” International Journal of Food Sciences and Nutrition, Vol. 59, No. 1, 2008, pp. 24-33. doi:10.1080/09637480701554103 